Dom. Cien., ISSN: 2477-8818

Vol. 5, núm.1., ene, 2019, pp. 218-241

\title{
Patología desencadenante en la enfermedad renal crónica
}

\author{
Pathology that triggers chronic kidney disease
}

\section{Patologia que desencadeia doença renal crónica}

\author{
María B. Gárate-Campoverde I \\ belen_garate@hotmail.com \\ Randy Mena-De La Cruz II \\ randy_mena@hotmail.com \\ Giancarlos C. Cañarte-Baque ${ }^{\text {III }}$ \\ gcanarte@gmail.com \\ Madeleine J. Sarmiento-Cabrera IV \\ sarmiento@gmail.com \\ Derlin A. Delgado-Janumis V \\ delgado_25@yahoo.com \\ María F. Santana-Reyes VI \\ mafercita_92@live.com
}

Recibido: 10 de octubre de 2018 * Corregido: 30 de octubre de 2018 * Aceptado: 30 de noviembre de 2018

I. Médica de la Universidad Católica de Santiago de Guayaquil; Médico Auditor, Coordinación provincial de prestaciones del seguro de salud del Guayas, Guayaquil, Ecuador.

II. Médico Cirujano de la Universidad Técnica de Manabí, Manta, Ecuador.

III. Médico de la Universidad Católica de Santiago de Guayaquil; Médico General Asistencial en Servicio de Cirugía General de Hospital Regional Dr. Verdi Cevallos, Portoviejo, Ecuador.

IV. Médica de la Universidad Católica de Santiago de Guayaquil, Guayaquil, Ecuador.

v. Magister en Salud Publica mención Gerencia de los Servicios de Salud; Licenciada en Enfermería; Docente de la Universidad Estatal del Sur de Manabí, Manta, Ecuador.

VI. Licenciada en Enfermería, Universidad Técnica de Manabí, Manta, Ecuador. 


\section{Resumen}

La enfermedad renal crónica (ERC) se reconoce como un problema de salud pública a nivel mundial, debido a su incidencia y prevalencia cada vez más alta. Es de patología muy desencadenante presenta manifestaciones clínicas muy variadas en los pacientes, es enfermedad crónica degenerativa, afecta la mayoría de los órganos causando alteraciones estructurales y funcionales del riñón, es una enfermedad no trasmisible y esta estrechamente ligada a otras enfermedades, como las metabólicas (diabetes, obesidad, dislipidemia, hiperuricemia) y cardiovasculares (hipertensión, hipertrofia ventricular izquierda, insuficiencia cardíaca, ateroesclerosis, etc.), también hay otros factores de riesgo, entre los que destacan los de susceptibilidad, como: edad, historia familiar, raza, bajo nivel educativo y económico, así como los factores indicadores, precursores de la enfermedad. Por lo tanto es de primordial interés atender las complicaciones que sobrelleva la disfunción renal; su comportamiento crónico e irreversible, es así como su potencial letalidad requiere un nivel avanzado de complejidad técnica para su manejo, dado que el diagnóstico de una enfermedad crónica impacta al enfermo y a su entorno afectivo inmediato, más allá de las meras implicaciones biomédicas.

Palabras claves: Enfermedad Renal Crónica; Enfermedades Metabólicas; Enfermedades Cardiovasculares; Factores de Riesgo; Técnica para su Manejo. 


\begin{abstract}
Chronic kidney disease (CKD) is recognized as a public health problem worldwide, due to its increasingly high incidence and prevalence. It is very triggering pathology presents clinical manifestations very varied in patients, is a chronic degenerative disease, affects most organs causing structural and functional alterations of the kidney, is a non-communicable disease and is closely linked to other diseases, such as metabolic ( diabetes, obesity, dyslipidemia, hyperuricemia) and cardiovascular (hypertension, left ventricular hypertrophy, heart failure, atherosclerosis, etc.), there are also other risk factors, among which are those of susceptibility, such as: age, family history, race, low educational and economic level, as well as the indicator factors, precursors of the disease. Therefore, it is of primordial interest to attend to the complications that the renal dysfunction bears; its chronic and irreversible behavior, is how its potential lethality requires an advanced level of technical complexity for its management, given that the diagnosis of a chronic disease impacts the patient and his immediate affective environment, beyond the mere biomedical implications.
\end{abstract}

Key words: Chronic Kidney Disease; Metabolic Diseases; Cardiovascular Diseases; Risk Factors; Technique For Its Management.

\footnotetext{
229 Vol. 5, núm. 1, enero 2019, pp. 218-241 María B. Gárate-Campoverde; Randy Mena-De La Cruz; Giancarlos C. Cañarte-Baque; Madeleine J. Sarmiento-Cabrera; Derlin A. Delgado-Janumis; María F. Santana-Reyes
} 


\section{Introducción.}

La enfermedad renal crónica (ERC) es una de las patologías de más alta prevalencia a nivel mundial. En la actualidad se estima que el 11,5\% de la población adulta en los Estados Unidos padece de ERC y esta incrementa la prevalencia de enfermedad cardiovascular, Coresh, et al. (2007) y Weiner, et al. (2004).

Se diagnostica cuando existe evidencia de daño renal por más de tres meses, y se divide en cinco estadios, dependiendo del volumen de filtración glomerular.2 La ERC es una condición relativamente común ( 1 de cada 10 personas la padecen), generalmente cursa asintomática y con frecuencia su progreso a insuficiencia renal terminal pasa inadvertido, por lo que es vital su prevención y detección en etapas tempranas. Evans, y Taal, (2011).

La enfermedad renal crónica (ERC) es una enfermedad progresiva, no trasmisible y estrechamente ligada a otras enfermedades, como las cardiovasculares (ECV) y la diabetes mellitus (DM). A pesar de su carácter prevenible, su incidencia está en aumento en todo el mundo, principalmente en épocas de globalización, en las que se han introducido nuevos factores de riesgo derivados de la producción y consumo, riesgos que se han sumado a la pobreza e inequidad social y sanitaria. (Lopera-Medina, M;.2016).

La Enfermedad Renal Crónica (ERC) es una patología con manifestaciones clínicas muy variadas, que afecta a la mayor parte de órganos y sistemas, que son reflejo de las complejas funciones que el riñón desempeña y las importantes consecuencias que comporta la disfunción renal. (Sierra, et al; 2007).

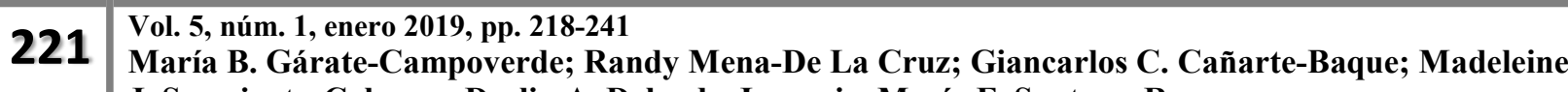
J. Sarmiento-Cabrera; Derlin A. Delgado-Janumis; María F. Santana-Reyes
} 
De acuerdo con la información del National Kidney Foundation Kidney Disease Outcomes Quality Initiative (NKF-K/ DOQI), la enfermedad renal crónica (ERC) se define como:

- Presencia de marcadores de daño renal por más de 3 meses (anormalidades en la composición sanguínea, urinaria, en estudios por imagen o anatomía patológica), indicando alteraciones estructurales o funcionales del riñón.

- Filtrado glomerular $(\mathrm{FG})<60 \mathrm{ml} / \mathrm{min}$ por más de 3 meses, con otros signos de daño renal o sin ellos

Se la considera una entidad irreversible y progresiva. El riñón es susceptible de ser afectado por múltiples situaciones. Una vez que se produce la lesión y se desarrolla ERC, se desencadenan distintos mecanismos que inducen deterioro progresivo de la función renal, con probabilidad de evolucionar a la pérdida funcional. (Russomando, 2014).

Recientes estudios epidemiológicos a nivel mundial han identificado variables que son predictivas de ERC, conocidas como factores desencadenantes, y factores que aumentan el riesgo de progresión de la enfermedad, conocidos como factores perpetuadores. La diabetes mellitus y las enfermedades cardiovasculares figuran como los principales factores desencadenantes y perpetuadores de la ERC, tanto en países desarrollados como en los subdesarrollados. Se estima que cerca de un $70 \%$ de los casos de enfermedad renal terminal, obedecen a las mencionadas patologías. El envejecimiento de la población, factores genéticos, la etnicidad, sexo, factores socioeconómicos, el bajo peso al nacer, la malnutrición infantil y la exposición a nefrotoxinas, entre otros, se mencionan como posibles factores desencadenantes o multiplicadores. (Rivera y Méndez; 2016).

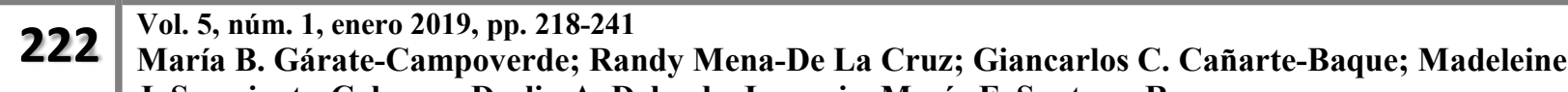
J. Sarmiento-Cabrera; Derlin A. Delgado-Janumis; María F. Santana-Reyes
} 
En la actualidad el paciente con una enfermedad renal avanzada se incluye en lista de espera antes de entrar en hemodiálisis y así asegurar una mejor forma física ante la intervención quirúrgica. (De Oliveira et al; 2012).

Las consecuencias a nivel económico y social son muy importantes. La tasa de morbilidad y mortalidad de los pacientes en tratamiento renal sustitutivo es muy elevada, aunque una de las razones principales se debe al aumento de edad de la población y, en consecuencia, al hecho de que esta mayoría de afectados son pacientes multipatológicos. (Martín de Francisco, 2010).

Actualmente se está prestando un gran interés a la detección precoz de la «enfermedad renal oculta» en la población general, a partir de la estimación del FG. Para ello se han desarrollado una serie de ecuaciones matemáticas basadas en la creatinina sérica (Crs). Así, según el grado de FG, la enfermedad renal crónica (ERC) se divide en varios estadios, considerando ERC estadio 3 a un FG entre 60 y $30 \mathrm{ml} / \mathrm{min} 6$. Esta clasificación se ha considerado aplicable a toda la población. (Heras et al, 2008).

\section{Metodología.}

En el presente trabajo científico tiene una visión específica, Se ha cumplido con una revisión de literatura en diferentes bases de datos se hizo la revisión más próxima. Para la realización de la actual investigación, se consideró, información confiable sobre trabajos figurados, versados en este tema, en los que se estudia el impacto en la calidad de vida y los distintos problemas relacionados con la enfermedad renal crónica, se utilizaron informaciones bibliográficas, se la obtuvo de libros electrónicos, consultas electrónicas, artículos científicos y revistas.

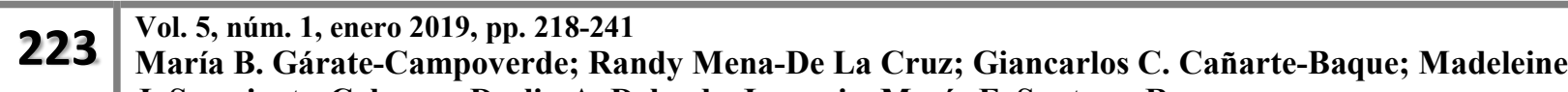




\section{Resultados.}

\section{La enfermedad renal crónica Definición}

La enfermedad renal crónica (ERC) afecta a un porcentaje cada vez mayor de la población debido a que las principales causas de ERC residen en trastornos de alta prevalencia como la diabetes, hipertensión y enfermedad cardiovascular, teniendo en cuenta, además, el aumento de la esperanza de vida y el envejecimiento progresivo de la población. La evolución natural de la ERC es la progresión hacia la enfermedad renal crónica terminal y la consiguiente necesidad de tratamiento sustitutivo mediante diálisis o trasplante renal. (Otero et al; 2005).

La enfermedad renal crónica (ERC) es una de las enfermedades no trasmisibles que, a pesar de ser prevenibles, están ocasionando mayor mortalidad en mundo. En esta se incluye un grupo de enfermedades heterogéneas cuyas manifestaciones y curso clínico dependen de la causa y el tipo de afectación, la gravedad, la tasa de progresión y las comorbilidades. (Levey et al, 2007).

La enfermedad renal crónica (ERC) es un problema de salud pública. La manifestación más grave de la ERC, la insuficiencia renal crónica terminal (IRCT) subsidiaria de tratamiento sustitutivo mediante diálisis o trasplante renal, presenta una incidencia y una prevalencia crecientes desde hace dos décadas. Se estima que por cada paciente en un programa de diálisis o trasplante puede haber 100 casos de ERC menos grave en la población general. Por un lado, estos casos constituyen la base de los pacientes que llegarán más tarde a una ERC avanzada. Por otro lado, estos pacientes tienen un riesgo cardiovascular elevado y sufren una morbimortalidad por eventos cardiovasculares que, probablemente, tenga un impacto en la salud mayor que la evolución hacia la necesidad de tratamiento renal sustitutivo. (Soriano, 2004).

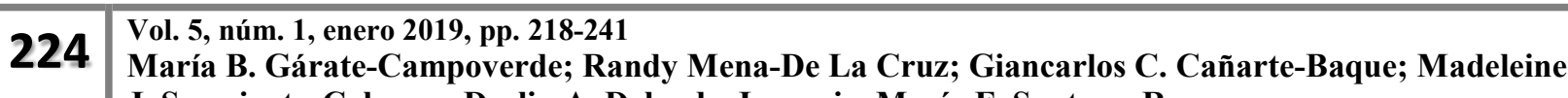


Desde un punto de vista clínico, existen diversos factores que influyen poderosamente en la progresión de las enfermedades renales, debe tenerse en cuenta que estos factores actúan de manera combinada, potenciándose sus efectos nocivos sobre las estructuras y la función renal. Se reconocen como causas más frecuentes de la insuficiencia renal crónica: la diabetes mellitus, la hipertensión arterial, las glomerulopatías y las enfermedades obstructivas renales. Los síntomas de ECR son muy variados y pocas veces claros. Algunos pacientes no muestran síntomas por mucho tiempo. Produce lesiones graves antes de que la persona sospeche que está enferma. La mayoría son de índole general: debilidad, irritabilidad, dolor de cabeza, orinar constantemente durante la noche, mareos, náuseas, vómitos, picazón, el cuerpo se hincha, hay falta de aire, pérdida del apetito, fatiga, lentitud y calambres. (Gonzáles et al, 2011).

Con el avance de la enfermedad, la piel se vuelve de color terroso, seca y con tendencia a descamarse, con manchas y aumento de la presión arterial, aliento con olor a orina, dificultad para respirar, mucho sueño durante el día y dificultad para dormir durante la noche. (Sellarés, Martín; 2002).

Recientemente, la National Kidney Foundation estadounidense ha propuesto a través de las guías de práctica clínica K/DOQI una definición y una clasificación de la ERC con los objetivos, entre otros, de aunar criterios y facilitar de forma sencilla y práctica el diagnóstico precoz de la enfermedad independientemente de la causa original. La ERC se define como una disminución de la función renal, expresada por un filtrado glomerular (FG) o por un aclaramiento de creatinina estimados $<60 \mathrm{ml} / \mathrm{min} / 1,73 \mathrm{~m} 2$, o como la presencia de daño renal de forma persistente durante al menos 3 meses. El daño renal se diagnostica habitualmente mediante marcadores en vez de por una biopsia renal por lo que el diagnóstico de ERC, ya se establezca por un FG disminuido o por

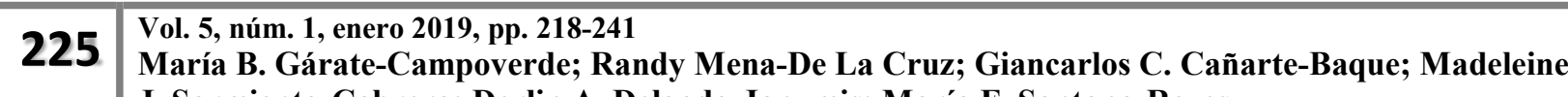
J. Sarmiento-Cabrera; Derlin A. Delgado-Janumis; María F. Santana-Reyes
} 
marcadores de daño renal, puede realizarse sin conocimiento de la causa. El principal marcador de daño renal es una excreción urinaria de albúmina o proteínas elevada. (Soriano; 2004).

En la nomenclatura de la nefrología mundial actualmente utilizada, el término insuficiencia renal crónica ha quedado fuera de uso y reemplazado por enfermedad renal crónica (ERC). Algunas asociaciones aun lo usan, principalmente en Latinoamérica, para referirse a la alteración en la función renal, en la cual la tasa de filtración glomerular (TFG) es menor a $60 \mathrm{ml} / \mathrm{min}$. (Henao; 2018).

\section{La enfermedad renal y patología}

Los riñones filtran los desechos de la sangre y regulan otras funciones del organismo. Estos purifican la sangre al quitarle el exceso de líquidos, minerales y productos de desecho, además de producir hormonas que mantienen la salud de los huesos y la sangre. La actividad de los riñones puede verse afectada por diferentes causas, muchas de ellas relacionadas con la calidad de vida y estilos de vida que asumen las personas. Cuando los riñones son incapaces de cumplir las funciones anteriormente mencionadas estamos en presencia de una Enfermedad Renal Crónica. La mayoría de las veces se presenta lentamente, no tiene cura y llega hasta una etapa terminal en la que el enfermo necesita un tratamiento renal sustitutivo del tipo de la Hemodiálisis, Diálisis Peritoneal o Trasplante Renal. (Gonzáles et al; .2011).

Una incidencia y una prevalencia crecientes en las últimas décadas. La visión epidemiológica de la ERC ha cambiado notablemente. Restringida inicialmente a patología

de incidencia baja como las enfermedades renales clásicas, en la actualidad la ERC afecta a un porcentaje significativo de la población debido fundamentalmente a que sus causas principales

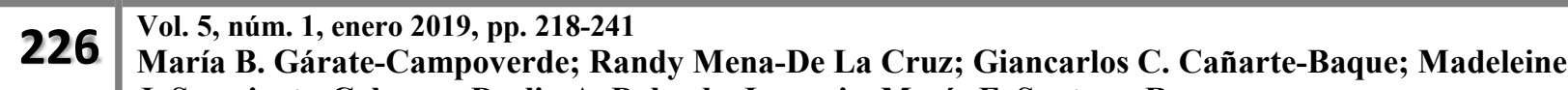
J. Sarmiento-Cabrera; Derlin A. Delgado-Janumis; María F. Santana-Reyes
} 
residen en trastornos de alta prevalencia como el envejecimiento, la hipertensión arterial (HTA), la diabetes y la enfermedad vascular. (Soriano; 2004).

Una idea generalizada asume que la ERC es una patología rara y compleja, pero la realidad es que en sus fases tempranas es frecuente y de fácil tratamiento. Sólo una pequeña proporción de enfermos evoluciona hacia la insuficiencia renal terminal con sus complicaciones asociadas y necesidad de tratamiento renal sustitutivo. Esta evolución hacia la pérdida de función renal tiene un curso progresivo, en el que podemos influir mediante una actuación precoz sobre sus principales causas: hipertensión arterial (HTA) y diabetes mellitus. El control de estas dos afecciones debe ser estricto y adecuado a las recomendaciones de las guías en vigor, no sólo para minimizar su progresión y tratar las complicaciones inherentes a la insuficiencia renal, sino también para reducir el riesgo vascular asociado a la ERC. El descenso de la función renal se asocia de forma significativa con un mayor riesgo cardiovascular. (Alcázar et al.; 2008).

La descripción clásica de la epidemiología de la ERC se ha restringido a la información sobre la IRCT en tratamiento renal sustitutivo procedente de los registros de diálisis y trasplante. Sucesivos informes del United States Renal Data System (USRDS) han documentado un aumento de la incidencia y prevalencia de IRCT en Estados Unidos en las últimas dos décadas. La incidencia actual se sitúa en los varones en 404 casos por millón de población y en las mujeres en 280 casos por millón de población. Se estima que la prevalencia de IRCT en tratamiento sustitutivo puede duplicarse en la década presente. En Europa, donde la incidencia de IRCT es claramente inferior a la de Estados Unidos, se ha detectado un incremento anual cercano al 5\% de pacientes en diálisis o con un trasplante renal. En nuestro país la incidencia de IRC en tratamiento sustitutivo se sitúa en 126

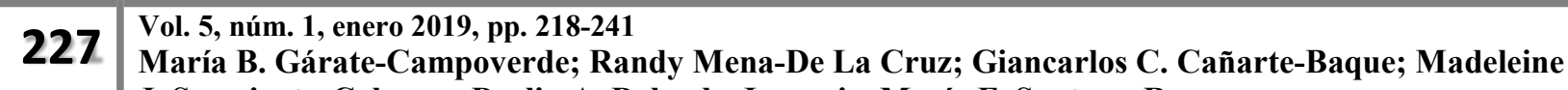


casos por millón de población, observándose también la especial repercusión de la edad avanzada, la HTA, la enfermedad vascular y la diabetes. (Soriano, 2004).

Las patologías que pueden producir lesión renal secundaria, como las enfermedades metabólicas (diabetes, obesidad, dislipidemia, hiperuricemia) y cardiovasculares (hipertensión, hipertrofia ventricular izquierda, insuficiencia cardíaca, ateroesclerosis, etc.), así como promover el abandono de hábitos tóxicos (tabaquismo, consumo de drogas ilícitas, abuso de antiinflamatorios, etc.) Las alteraciones cardiovasculares y metabólicas pueden producir daño renal a través de mecanismos hemodinámicos (estimulación simpática y del sistema renina-angiotensinaaldosterona) o por medio de la inducción de inflamación, estrés oxidativo y liberación de citoquinas proinflamatorias. (Russomando; 2014).

\section{Detección precoz}

Es la identificación del daño en estadios tempranos, lo que permite realizar un tratamiento efectivo para evitar o enlentecer la evolución de la ERC.

En primer lugar se deben identificar los pacientes con factores de riesgo:

- Diabetes

- Hipertensión

- Antecedentes personales de enfermedad coronaria, accidente cerebrovascular o enfermedades vasculares

- Tabaquismo

- Obesidad

- Exposición crónica a nefrotóxicos (incluidos los antiinflamatorios)

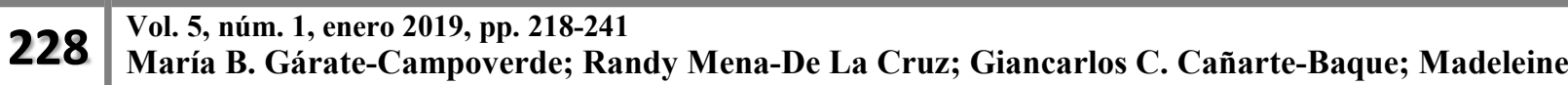
J. Sarmiento-Cabrera; Derlin A. Delgado-Janumis; María F. Santana-Reyes
} 
- Enfermedad de la vía urinaria

- Antecedentes familiares de nefropatía

- Enfermedades sistémicas

En todo paciente con algún factor de riesgo se debe realizar, por lo menos una vez al año:

- Examen de orina: densidad, sedimento (piuria, hematíes dismórficos, cilindros hemáticos, leucocitarios, céreos, anchos, etc.).

- Determinación de microalbuminuria y/o proteinuria.

- Función renal (clearence de creatinina medido o cálculo de la tasa de FG por las fórmulas) Russomando, (2014).

- Patología de la lesión renal que puede producir enfermedades metabólicas.

\section{Diabetes}

Es una de las epidemias de este siglo; se calcula su aumento mundial y que alcanzará para el año 2050, hasta 300 millones de personas, unido al cambio demográfico, se espera un incremento de los diabéticos fundamentalmente en la población $\geq 75$ años superior a $271 \%$ en mujeres y a $437 \%$ en hombres. (Boyle et al.; 2001).

La presencia de una ERC en pacientes con diabetes puede o no puede representar una Enfermedad Renal Diabética (ERD); existe mayor probabilidad de ERD si se detecta una macroalbuminuria, o la microalbuminuria se encuentra asociada a retinopatía diabética, en un paciente diabético 1 de $>10$ años de evolución. Su pesquisaje debe efectuarse después de 5 años de evolución en los diabéticos tipo 1 y en los diabéticos tipo 2 desde el mismo momento de su diagnóstico. (KDOQI-US; 2007).

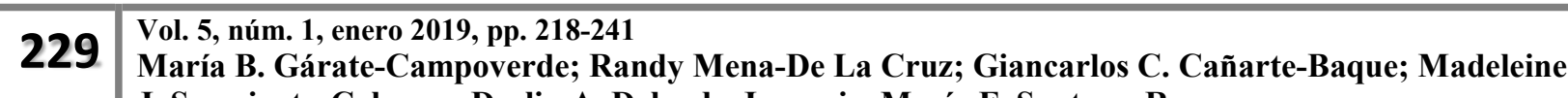


Las evidencias científicas demuestran que la presencia de complicaciones microangiopáticas cardíacas, renales y en el fondo de ojo, se presentan al no establecerse un estricto control metabólico en los diabéticos. (DCCT; 1995).

\section{Obesidad}

Ha sido determinada en varios estudios como un factor de riesgo para el desarrollo de ERC y progresión de la misma. Se ha visto en la población obesa mayor prevalencia de proteinuria, con el desarrollo de glomeruloesclerosis focal y segmentaria, como hallazgo en la histopatología renal de estos pacientes. La fisiopatología no es del todo conocida, se han propuesto teorías acerca de cambios hemodinámicos, aumento de sustancias vasoactivas, fibrogénicas, entre las que se incluyen la angiotensina II, insulina, leptina y factor de crecimiento transformante beta. (D’Achiardi; 2011).

\section{Dislipidemia}

Se ha reportado que el control metabólico, la hiperlipidemia y la acidosis metabólica se pueden relacionar con progresión de la ERC. El estudio SHARP proporcionó evidencia adecuada acerca de la eficacia y seguridad de disminuir los niveles de colesterol LDL en la incidencia de eventos ateroescleróticos mayores, en pacientes con ERC sin terapia de soporte renal. Aunque se encontró descenso en el deterioro de la FG calculada por las formulas MDRD4 y por COCKCROFT GAULT en pacientes tratados con simvastatina, no se alcanzó diferencia significativa en términos estadísticos, sin embargo, la estatina puede tener un efecto renoprotector en aquellos pacientes con ERC y enfermedad cardiovascular. (D'Achiardi; 2011).

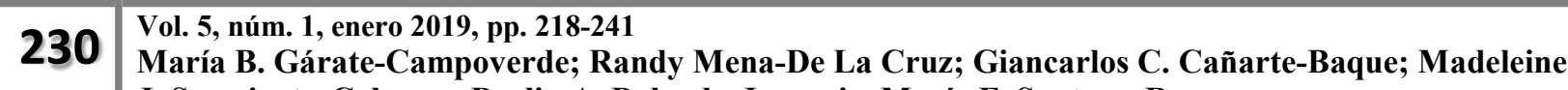
J. Sarmiento-Cabrera; Derlin A. Delgado-Janumis; María F. Santana-Reyes
} 


\section{Hiperuricemia}

La elevación de los niveles de ácido úrico se presenta en los pacientes con ERC, por disminución en la excreción urinaria. La hiperuricemia ha demostrado ser un factor de progresión de la enfermedad renal, en parte por disminución en la perfusión renal por estimulación en la proliferación de la musculatura en la arteriola aferente. Los estudios clínicos han mostrado resultados contradictorios, sin tener la capacidad de demostrar asociación directa que las cifras elevadas de ácido úrico aceleren el deterioro de la función renal, por lo anterior, se deber examinar con detenimiento la utilización de medicamentos con el propósito del control de hiperuricemia y no emplearlos de manera rutinaria. (D'Achiardi; 2011).

Patología de la lesión renal que puede producir enfermedad cardiovascular

Se ha reconocido una estrecha asociación entre la ERC y la enfermedad cardiovascular, de manera que la ERC contribuye al desarrollo de la patología cardiovascular y por su parte, la enfermedad vascular favorece la aparición y progresión de ciertas nefropatías. La ERC desde sus estadios iniciales está ligada a la enfermedad vascular sistémica y ambos trastornos comparten factores de riesgo y mecanismos de progresión. (KDOQI-US, 2002), (Marín et al.2006) y (Schrier; 2006).

\section{Hipertensión}

La HTA daña el riñón y el corazón, todo el árbol vascular, mucho más que por el solo efecto hemodinámico. Se adiciona la activación del sistema renina-angiotensina-aldosterona (SRAA) que activa los mediadores de la inflamación, el estrés oxidativo, el crecimiento celular y la acumulación

\footnotetext{
231 Vol. 5, núm. 1, enero 2019, pp. 218-241

María B. Gárate-Campoverde; Randy Mena-De La Cruz; Giancarlos C. Cañarte-Baque; Madeleine 
de matrix mesangial todos ellos efectos proinflamatorios y profibróticos. (Karalliedde y Viberti; 2006).

La HTA es a la vez causa y consecuencia de la ERC. Su prevalencia aumenta con la disminución de la función renal, alcanzando el $80 \%$ de pacientes en estadio 5. Están implicados varios mecanismos patogénicos. Estimulación del SRAA, hiperactividad simpática, expansión del volumen extracelular, disfunción endotelial, aumento del calcio intracelular, calcificaciones vasculares y posible enfermedad vascular renal. Los fenómenos que afectan la pared arterial son de dos tipos. El primero, la formación de placas de ateroma en la íntima, calcificadas con mayor frecuencia que en la población general. Se produce isquemia del territorio afectado y riesgo de oclusión por trombosis. El segundo, el engrosamiento, la infiltración y la calcificación de la media. Henao, (2018).

La elevación de las cifras de PA elevada a nivel sistémico, se han relacionado con un aumento de la presión a nivel del glomérulo, ocasionando alteraciones crónicas hemodinámicas de la arteriola aferente y llevando a un fenómeno conocido como hiperfiltración adaptativa. Esta es posiblemente la fase inicial de la ERC. D'Achiardi, (2011).

La pérdida de elasticidad arterial resultante provoca un incremento de la presión arterial sistólica y de la presión del pulso, sobrecarga cardiaca de presión, mala adaptación a la hipotensión y eventualmente hipoperfusión coronaria diastólica. Las alteraciones que tienen lugar en el corazón comprenden calcificaciones de las válvulas con disfunción valvular, calcificaciones y lesiones del sistema de conducción con arritmias, miocardiopatía con insuficiencia cardiaca y aterosclerosis coronaria con cardiopatía isquémica. La miocardiopatía urémica se produce por dos mecanismos, sobrecarga de presión y sobrecarga de volumen. La HTA y la falta de elasticidad de la aorta

\footnotetext{
232 Vol. 5, núm. 1, enero 2019, pp. 218-241 María B. Gárate-Campoverde; Randy Mena-De La Cruz; Giancarlos C. Cañarte-Baque; Madeleine J. Sarmiento-Cabrera; Derlin A. Delgado-Janumis; María F. Santana-Reyes
} 
condicionan una sobrecarga de presión que induce una HVI concéntrica. Hay un aumento de la presión sistólica, incremento de miofibrillas, engrosamiento de la pared y pocos cambios en el volumen ventricular. Henao, (2018).

Esta demostrada como evidencia científica, pero no es bien conocido ni comprendido por la comunidad médica, que la principal complicación de la ERC, es la enfermedad cardiovascular, y se comporta como un factor de riesgo independiente de agravamiento del daño renal y progresión a etapas más avanzadas de la ERC. (Elsayed et al.; 2007).

La prevalencia de hipertensión, al inicio de diálisis, (hasta 90\%), como factor mayor de enfermedad coronaria se expresa en que la hipertrofia ventricular izquierda existe en $75 \%$ de los mismos, y que, al menos, 35\% poseen una cardiopatía isquémica. (Levin; 2003).

\section{Hipertrofia ventricular izquierda}

La hipertrofia ventricular izquierda (HVI) es un factor de riesgo cardiovascular bien reconocido en la población general. En el curso de la enfermedad renal la HVI se desarrolla precozmente y su prevalencia aumenta de forma inversa al nivel de función renal. Es, además, la alteración cardíaca más frecuente en los pacientes con IRC terminal. La masa del ventrículo izquierdo aumenta progresivamente durante el tratamiento con diálisis, incluso en pacientes normotensos. Tras el trasplante renal, la HVI y la miocardiopatía dilatada mejoran, principalmente durante los dos primeros años, aunque no se normalizan. (Cases; 2004).

Los mecanismos implicados en el desarrollo de esta complicación son la sobrecarga de presión y la sobrecarga de volumen. La sobrecarga de presión es inducida por la hipertensión arterial (HTA), la rigidez de las grandes arterias (arteriosclerosis) o la estenosis aórtica, y conduce al

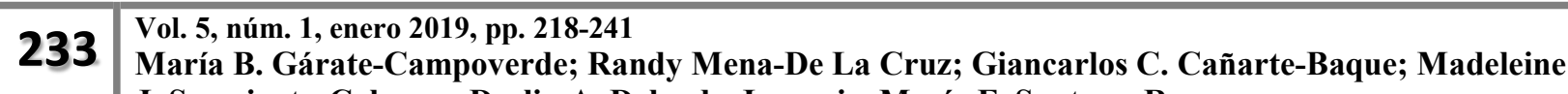
J. Sarmiento-Cabrera; Derlin A. Delgado-Janumis; María F. Santana-Reyes
} 
desarrollo de HVI concéntrica. La sobrecarga de volumen es inducida por la hipervolemia crónica, la anemia o la circulación hiperdinámica generada por el acceso vascular (fístula arteriovenosa), que se asocian con un aumento del gasto cardíaco y favorece el desarrollo de una HVI excéntrica. Para un mismo nivel de presión arterial, los pacientes renales presentan un índice de masa del ventrículo izquierdo (VI) superior al de pacientes no renales, indicando que otros factores contribuyen a su desarrollo. (Cases; 2004).

\section{Etiología y factores de riesgo}

La enfermedad renal crónica se ha transformado en un problema médico y de salud pública que ha adquirido proporciones epidémicas. De acuerdo con los datos de la Encuesta Nacional de Salud, la diabetes mellitus y la hipertensión arterial, respectivamente, son causas importantes de enfermedad renal, aunque también hay otros factores de riesgo, entre los que destacan los de susceptibilidad, como: edad, historia familiar, raza, bajo nivel educativo y económico, así como los factores indicadores, precursores de la enfermedad. Los de mayor importancia son los susceptibles de prevención, como las enfermedades crónicas, sistémicas y por toxicidad por fármacos. Flores, J. et al. (2009) y Dehesa, (2008).

Estadios evolutivos, según Venado, A. et al. (2005). La enfermedad renal crónica es progresiva y sigue un patrón constante que depende de su etiología y del propio paciente:

- Estadio 1: pacientes con diabetes y microalbuminuria con una TFG normal.

- Estadio 2: se establece por el daño renal asociado con la ligera disminución de la tasa de filtración gromerular entre 89 y $60 \mathrm{~mL} / \mathrm{min} / 1.73 \mathrm{~m}^{2}$. Por lo general, el paciente no tiene síntomas y el diagnóstico se establece de manera incidental. 
- Estadio 3: es la disminución moderada de la TFG entre 30 y $59 \mathrm{~mL} / \mathrm{min} / 1.73 \mathrm{~m} 2$. El estadio 3 se divide en dos etapas. La etapa temprana 3 a es la de pacientes con TFG entre 59 y $45 \mathrm{~mL} / \mathrm{min} / 1.73 \mathrm{~m} 2$ y la etapa tardía $3 b$ con TFG entre 44 y $30 \mathrm{~mL} / \mathrm{min} / 1.73 \mathrm{~m} 2$. Cuando la función renal disminuye, en el torrente sanguíneo se acumulan sustancias tóxicas que ocasionan uremia. Lo común es que los pacientes tengan síntomas y complicaciones típicas originadas por la hipertensión, anemia y alteraciones del metabolismo óseo.

- Estadio 4: se refiere al daño renal avanzado con disminución grave de la TFG entre 15 y $30 \mathrm{~mL} / \mathrm{min} / 1.73 \mathrm{~m} 2$. Pacientes con alto riesgo de complicaciones cardiovasculares.

- Estadio 5: o insuficiencia renal crónica terminal, la TFG cae por debajo de 15 $\mathrm{mL} / \mathrm{min} / 1.73 \mathrm{~m} 2$. En este estadio se requiere tratamiento sustitutivo.

Según Levey, et al. (2005), factores de riesgo de desarrollo de ERC, Incluye tres categorías principales:

1.- De susceptibilidad o predisponentes: Factores raciales, étnicos y genéticos (antecedentes familiares y/o personales de ERC, dializados o de enfermedad vascular ateroesclerótica cardioca cerebral perisférica, (infarto, angina, stroke en menores de 50 años), género masculino, bajo nivel de ingreso o educacional, bajo peso al nacer, edad avanzada, obesidad (= $30 \mathrm{~kg} / \mathrm{m} 2$ ), obesidad abdominal, sedentarismo e inactividad física, consumidores habituales de analgésicos o antiinflamatorios no esteroideos, riesgo laboral de exposición a nefrotóxicos: abonos, plomo, mercurio, entre otros.

2.- De iniciación o causales: HTA, DM 1 y 2, uropatías obstructivas, edad avanzada, enfermedades con lesión órgano específicas (hereditarias, infecciosas, sistémicas, tóxicas etcétera).

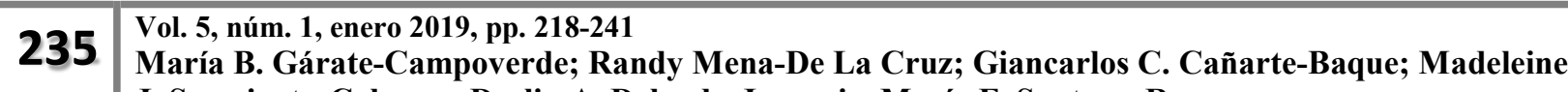
J. Sarmiento-Cabrera; Derlin A. Delgado-Janumis; María F. Santana-Reyes
} 
3.- Factores de riesgo modificables de progresión o complicaciones: tabaquismo, dislipidemias (hipercolesterolemia, hipertrigliceridemia, LDL-oxidado, Lp (a), microalbuminuria y/o proteinuria, anemia, factores protrombóticos (fibrinogeno etcétera), proteína $\mathrm{C}$ reactiva elevada.

\section{Bajo peso al nacer}

Ocurre con más frecuencia en minorías y comunidades con desventajas socio-económicas y se asocia en los adultos a ECV, HTA, DM, ERC. La hiperfiltración lomerular que las acompaña, se manifiesta tempranamente como microalbuminuria. Ahí radica la importancia de su control, posible erradicación y seguimiento mantenido con consulta sistemática desde la infancia para tratar precozmente la microalbuminuria y orientar estilos de vida saludable. (Pérez-Oliva et al.; 2008).

\section{Tabaquismo}

El tabaquismo es un factor de riesgo cardiovascular reconocido en la población general y un evidente predictor de aterosclerosis. La prevalencia del uso del tabaco en pacientes con enfermedad renal en sus diferentes estadios es similar a la de la población general. Sin embargo, con insuficiencia renal, el acúmulo de nicotina es más elevado. El tabaquismo se ha implicado como factor de riesgo cardiovascular en pacientes en diálisis desde hace años. Los datos recientes del USRDS Wave 2 Study otros indican que el tabaquismo se asocia con un mayor riesgo de desarrollo de insuficiencia cardíaca de novo, infarto de miocardio, vasculopatía periférica, y mortalidad en pacientes en hemodiálisis. (Cases; 2004).

Es un factor de riesgo vascular y renal modificable asociado a la progresión de la nefropatía en la DM tipo 1 y 2 y la ERC, viéndose implicado el óxido nítrico, incluso en su polimorfismo genético; de ahí la importancia de su supresión. (Pérez-Oliva et al.; 2008).

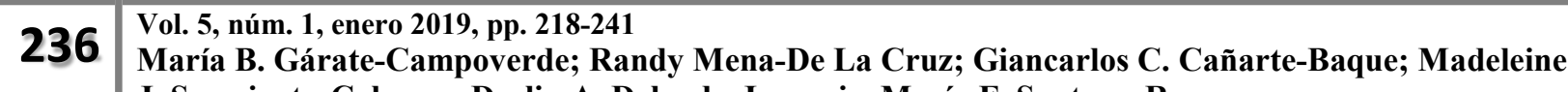
J. Sarmiento-Cabrera; Derlin A. Delgado-Janumis; María F. Santana-Reyes
} 
Estudios transversales han demostrado, en su mayor parte, una prevalencia aumentada de enfermedad cardiovascular en pacientes con IRC o en diálisis en fumadores que en no fumadores. En pacientes en diálisis favorece la rigidez arterial y el desarrollo de HVI. En estudios prospectivos el tabaco es un factor de riesgo de eventos coronarios en pacientes trasplantados. En estudios retrospectivos se asocia con un mayor riesgo de eventos cardiovasculares y muerte. El riesgo es dosis-dependiente y se reduce tras 5 años de su abandono. (Cases; 2004).

Dislipidemias (Hipercolesterolemia, hipertrigliceridemia, LDL-oxidado, Lp (a)

Como clásico factor de enfermedad cardiovascular y asociado también a la ERC deben ser controladas y erradicadas con ejercicio, dieta y medicamentos. (Pérez-Oliva et al.; 2008).

\section{Anemia}

La anemia en la ERC se caracteriza por ser del tipo normocítica y normo crómica; el recuento de reticulocitos es bajo para la anemia y la fracción eritroide de la medula se encuentra hipoplasica, sin que exista interferencia con las otras líneas hematológicas (leucocitos y plaquetas). Puede detectarse en estadios tempranos de la enfermedad, haciéndose más severa a medida que empeora la función renal. El déficit en la secreción de

EPO es el principal mecanismo patogénico. (Henao; 2018).

Otros factores múltiples contribuyen al desarrollo de la anemia renal, la vida media de los glóbulos rojos está acortada, algunas moléculas del grupo de poli aminas, como la espermina y espermidina, se comportan como toxinas urémicas; inhibiendo la eritropoyesis, aunque estas teorías en los últimos estudios han perdido validez debido a su dificultad para reproducir estos hallazgos,

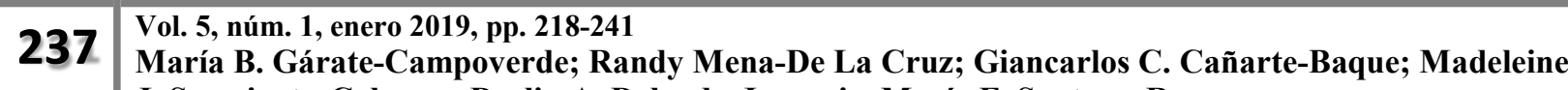
J. Sarmiento-Cabrera; Derlin A. Delgado-Janumis; María F. Santana-Reyes
} 
por lo cual el papel de estas sustancias inhibidoras en la etiología de la anemia se a minimizado. Por otra parte, se puede presentar déficit de hierro y vitaminas, como el ácido fólico y la vitamina B, así como pérdidas hemáticas, intoxicación por aluminio y fibrosis de la médula ósea secundaria a hiperparatiroidismo. La anemia contribuye al desarrollo de insuficiencia cardiaca en la uremia crónica. (Henao; 2018).

\section{Conclusiones.}

La enfermedad renal crónica con patología desencadenante presenta manifestaciones clínicas muy variadas, es enfermedad crónica degenerativa, afecta la mayoría de los órganos desordenando las complejas funciones que cumple el riñón, por lo tanto es de primordial interés atender las complicaciones que sobrelleva la disfunción renal; por consiguiente la ERC se presenta como un problema de salud de suma importancia en crecimiento, dada esta situación la enfermedad impone un importante desafío, dada la gravedad del problema los sistemas de salud, la comunidad médica enfrenten lo complicado de la patología, en la que se debe educar a la población, a fin de buscar la adhesión a las recomendaciones puede asegurar o no un resultado eficaz en cada caso particular, manifestar a la sociedad que la enfermedad renal crónica es frecuente, dañina, prevenible y en cierto modo curable. Existe una serie de guías de práctica clínica sobre la evaluación, clasificación y estratificación de la ERC, con el objetivo de aunar criterios y prevenir o retrasar las complicaciones de la enfermedad mediante el diagnóstico precoz y manejo apropiado de la enfermedad, independientemente de la causa original. Esta claro el padecimiento crónico degenerativo que se asocian con una serie de complicaciones como en el caso del funcionamiento de los riñones, los diversos factores vinculados a las condiciones de vida de las personas que la padecen tiene que

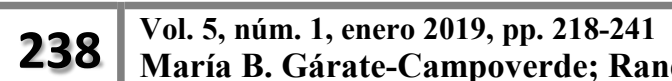
María B. Gárate-Campoverde; Randy Mena-De La Cruz; Giancarlos C. Cañarte-Baque; Madeleine J. Sarmiento-Cabrera; Derlin A. Delgado-Janumis; María F. Santana-Reyes
} 
aprender a vivir con los síntomas y complicaciones que ocasionan padecimientos como angustia, tristeza, etc.

\section{Referencias Bibliográficas.}

Alcázar, R., Egocheaga, I., Orte, L., Lobos, J., González, E., Álvarez, F., Górriz, J., Navarro, J. y Martín de Francisco, A., (2008), Documento de consenso SEN-semFYC sobre la enfermedad renal crónica, Nefrología 2008, 28 (3) 273-282 http://www.senefro.org

Boyle, J., Honeycutt, A., Narayan, K., Hoerger, T., Geiss, L., Chen, H., Thompson, T., (2001), Projection of diabetes burden through 2050: impact of changing demography and disease prevalence in the U.S. Diabetes Care, 24(11): 1936-1940.

Cases, A., (2004), Otros factores de riesgo cardiovascular y renal. Hipertrofia del ventrículo izquierdo. Fibrilación auricular. Tabaquismo. Obesidad. Factores emergentes de riesgo cardiovascular: Homocisteína. Proteína C reactiva. Fibrinógeno, Nefrología, Volumen 24, Suplemento $\mathrm{N}^{\mathrm{o}}$ 6, Capítulo 5, Páginas $0-235$.

Coresh, J., Selvin, E., Stevens, L.A., et al. (2007), Prevalence of chronic Kidney disease in the United States, JAMA, 298(17): 2038-2047.

D’Achiardi Rey, R., Vargas, J.G., Echeverri, J.E., Moreno, M., Quiroz, G., (2011), Factores de riesgo de enfermedad renal crónica, revista facultad medicina, Volumen 19, No. 2.

Dehesa, L., (2008), Enfermedad renal crónica, definición y clasificación, El Residente 2008; 3: 73 78.

De Oliveira, A.M., De Souza, S.R., Lopes, B. y Novaes, C., (2012), El enfermero asistencial y educador en una unidad de trasplante renal: un desafío, Enferm. Glob, 11(27).

DCCT, (1995), Effect of intensive therapy on the development and progression of diabetic nephropathy in the Diabetes Control and Complications Trial, The Diabetes Control and Complications (DCCT) Research Group, Kidney Int. 47: 1703-20.

Elsayed, E., Tighiouart, H., Griffith, J., Kurth, T., Levey, A., Salem, D., Sarnak, M., Weiner, D., (2007), Cardiovascular disease and subsequent kidney disease, Arch Intern Med. 167(11): $1130-1136$.

Evans, P.D. y Taal, M.W., (2011), Epidemiology and causes of chronic kidney disease, Medicine, 39: 402-406.

Flores, J. et al. (2009), Enfermedad renal crónica: clasificación, identificación, manejo y complicaciones. Rev Méd Chile 2009; 137:137-177.

Gonzáles, Y., Herrera, L. F., Romero, J. L. \& Nieves, Z. (2011), Características del estado emocional en pacientes con enfermedad renal crónica, Revista PsicologiaCientifica.com, 
13(20), Disponible en: http://www.psicologiacientifica.com/enfermedad-renal-cronicapacientes-estado-emocional

Henao Velásquez, Carlos, (2018), Enfermedad renal crónica, Nefrología Básica 2, Capítulo 23. Disponible en: http://asocolnef.com/wp-content/uploads/2018/03/Cap23.pdf

Heras, M., Fernández, M., Sánchez, L., Guerrero, M., Muñoz, A., Macías, M., Molina, A., Prado, F. y Álvarez, F., (2008). Ancianos con enfermedad renal crónica: ¿cuál es su evolución al cabo de un año?, Nefrología 2008; 28 (3) 325-328. http://www.senefro.org

Karalliedde, J., Viberti, G., (2006), Evidence for renoprotection by blockade of the reninangiotensin-aldosterone system in hypertension and diabetes, J Hum Hypertens, 20(4): 23953.

KDOQI-US, (2007), Clinical Practice Guidelines and Recommendations for Diabetes and Chronic Kidney Disease. Disponible en: www. Kdoqi

KDOQI-US, (2002), Clinical Practice Guidelines for Chronic Kidney Diseases: Evaluation, Classification, and Stratification. National Kidney Foundation: K/DOQI, Am J Kidney Dis; 39 Suppl 1: S1-S266.

Levey, A., Atkins, R., Coresh, J., Cohen, E., Collins, A., Eckardt, K., et al., (2007), Enfermedad renal crónica como problema global en salud pública: Abordajes e iniciativas, Propuesta de la Kidney Disease Improving Global Outcomes, Revista Kidney International, 2007, 3: 23245.

Levey, A., Eckardt, K., Tsukamoto, Y., Levin, A., Coresh, J., Rossert, J., De Zeeuw, D., Hostetter, T., Lameire, N., Eknoyan, G., (2005), Definition and classification of chronic kidney disease: A position statement from Kidney Disease, Improving Global Outcomes (KDIGO), Kidney Int. 2005, 67: 2089-2100.

Levin, A., (2003), Clinical epidemiology of cardiovascular disease in chronic kidney disease prior to dialysis. Semin Dial. 16(2): 101-5.

Lopera-Medina MM. (2016), La enfermedad renal crónica en Colombia: necesidades en salud y respuesta del Sistema General de Seguridad Social en Salud, Rev. Gerenc. Polit. Salud, 15(30): 212-233. http://dx.doi.org/10.11144/Javeriana.rgyps15-30.ercc

Martín de Francisco, A.L., (2010), El futuro del tratamiento de la enfermedad renal crónica. Nefrología, 30(1).

Marín, R., Goicoechea, M., Gorostidi, M., Cases, A., Díez, J., Escolar, G., et al., (2006), Guía de la Sociedad Española de Nefrología sobre riñón y enfermedad cardiovascular, Versión abreviada. Nefrología, 26(1): 31-44.

Otero, A., Gayoso, P., García, F., De Francisco, A., (2005), Epidemiology of chronic renal disease in the Galician population: Results of the pilot Spanish EPIRCE study, Kidney Int 68 suppl 99: S16-S19. 
Pérez-Oliva, J., Herrera, R., Almaguer, M., (2008), ¿Como mejorar el manejo de la enfermedad renal crónica? consideraciones y recomendaciones practicas, Revista habana ciencia médica La Habana, Volumen VII, No. 1.

Rivera-Chavarría, Ana, y Méndez-Chacón, Ericka, (2016), Mortalidad y egresos hospitalarios por enfermedad renal crónica compatibles con enfermedad crónica de causas no tradicionales, Costa Rica, Acta Médica Costarricense, (C) 2016, Colegio de Médicos y Cirujanos de Costa Rica, ISSN 0001-6012/2016/58/1/4-14

Russomando, S., (2014), Enfermedad renal crónica, revisión de tema y recomendaciones OSECAC, Cli-110, v2-14, Página 1-12. Disponible http://www.osecac.org.ar/documentos/guias_medicas/GPC\%202008/Clinica-Medica/Cli$110 \% 20$ Enfermedad $\% 20$ renal $\% 20 \mathrm{cr} \% \mathrm{C} 3 \% \mathrm{~B} 3$ nica_v2-14.pdf

Sellarés, V.L., Martín, M.L., (2002), Insuficiencia renal crónica. Manual de Nefrología, 2da ed. Madrid: Harcourt, p. 173-192.

Sierra, P., Monsalve, C., Comps, O., Andrés, E., (2007), Valoración preoperatoria del paciente con Enfermedad renal crónica, Societat Catalana d'Anestesiologia, Reanimació i Terapia del Dolor, Disponible en: http://www.scartd.org/arxius/erc231006.pdf

Schrier, R., (2006), Role of Diminished Renal Function in Cardiovascular Mortality. Marker or Pathogenic Factor? J Am Coll Cardiol, 47: 1-8.

Soriano Cabrera, S., (2004), Definición y clasificación de los estadios de la enfermedad renal crónica, Prevalencia, Claves para el diagnóstico precoz, Factores de riesgo de enfermedad renal crónica, NEFROLOGÍA, Volumen 24, Suplemento $\mathrm{N}^{\mathrm{0}}$ 6, Capítulo 2, páginas 0-235. Disponible en: http://www.revistanefrologia.com/es-definicion-clasificacion-los-estadiosenfermedad-renal-cronica-prevalencia-claves-el-articulo-X0211699504030666

Venado, A. et al. (2005), Insuficiencia renal crónica. Unidad de Proyectos Especiales, Universidad Nacional Autónoma de México, México, 1-31.

Weiner, D.E., Tighiouart, H., Amin, M.G., et al. (2004), Chronic Kidney disease as a risk factor for cardiovascular disease and all-cause mortality, J Am SocNephrol, 15(5): 1307-1315. 